\title{
Exploit and ignore the consequences: a mother of planetary issues
}

\author{
Khaled Moustafa*
}

\begin{abstract}
Many environmental and planetary issues are due to an exploitation strategy based on exploit, consume and ignore the consequences. As many natural and environmental resources are limited in time and space, such exploitation approach causes important damages on earth, in the sea and maybe soon in the space. To sustain conditions under which humans and other living species can coexist in productive and dynamic harmony with their environments, terrestrial and space exploration programs may need to be based on 'scrutinize the consequences, prepare adequate solutions and then, only then, exploit'. Otherwise, the exploitation of planetary resources may put the environmental stability and sustainability at a higher risk than it is currently predicted.
\end{abstract}

Keywords: environmental sustainability; Earth mass; oil extraction; fossil fuel; lunar exploration; gravitational force.

\section{Main Text}

I thank Dr. Unice Oleander (Oleander 2016) for the valuable comment on my paper "Oil, Earth mass and gravitational force" (Moustafa 2016). Dr. Oleander is right to say that it is impracticable to manipulate every gravitational body in the solar system and, thus, proposes to pay more attention to proximal celestial bodies, in particular the Moon. Indeed, the relative proximity between the Moon and Earth ( $\left.357,000 \mathrm{~km}^{*}\right)$, compared to other celestial bodies, makes that any substantial change in the mass of any of them (Earth or Moon) might have mutual repercussions on both of them, based on reciprocal gravitational interactions. The recent description of gravitational waves and the fact that such waves propagate freely through the Earth (Abbott et al. 2016) might confirm mutual gravitational repercussions between planets and celestial bodies in the universe. Mining the Moon, however, as a potential approach to maintain mass homeostasis between Earth and Moon was not, and indeed is not, my suggested approach. I am rather skeptical about the feasibility and the efficacy of such an approach

\footnotetext{
${ }^{*}$ http://nssdc.gsfc.nasa.gov/planetary/factsheet/moonfact.html
} 
from viable, economical, logistical, and sustainable perspective. I rather do call to minimize the reliance on fossil fuels and to develop cleaner and safer energy alternatives, based on the solar energy relatively abundant on Earth.

Although the percentage of the extracted fossil fuels' mass seems to be small compared to the global Earth's mass as previously highlighted (Moustafa 2016), and beyond technical, financial and logistical challenges for mining the Moon, it might be much wiser to solve Earth problems on Earth rather than to export them to the Moon and create new problematic challenges in the space. Moreover, the lunar exploitable resources might not be as abundant as fossil fuels on Earth to eventually counterbalance the potential loss of terrestrial mass by the extraction of equivalent lunar material and bring it to Earth. It is hardly imaginable that we could sustain, control and bring trillion tons of lunar materials to Earth. Mining the moon, however, for rare minerals or gases in small quantities might be envisioned in the future for specific purposes that would not require huge amounts of lunar resources. Future scientific advancements in remote and robotic technologies would allow such endeavors to be reachable. In fact, China (Zheng et al. 2008), Japan (Matsumoto et al. 2006) and many other space agencies have already developed exploration strategies outlining rationales and goals for exploring the Moon and other destinations (Schaffer 2008). The analysis of the lunar geological archive for example might help advance the comprehension of the origin and evolution of the Solar System (Crawford and Joy 2014). The abundance of the Helium-3 in the lunar regolith layer could also be a valuable resource as a potential fusion fuel on Earth ( $\mathrm{Fa}$ and Jin 2007). Nevertheless, mining the moon at large scales for extracting and bringing trillion tons of lunar materials on Earth will be extremely challenging, risky, costly and possibly conflictual for 'proprietary and priority' rights between powerful nations. Additionally, a lunar exploration philosophy based on conquer, exploit, and ignore the consequences would be disastrous at the long-term (Williamson 2005 9048).

In my opinion, if comparable efforts and investments devoted to lunar mining infrastructure would be devoted to developing alternative energy sources on Earth (i.e. solar and renewable energy alternatives), the outcome would be more rewarding than to spend a lot of efforts and money for uncertain remote outcomes. We might need new efficient development and sustainable policies for terrestrial resources (Dittmar 2014) before competing or investing massively to explore and deplete other planetary resources that may produce incontrollable effects or adverse consequences. Exploration and exploitation strategies of environmental resources may need to be based on a multi-criteria decision basis that takes into account short and long-term impacts (Huang et al. 2011), so that the 
environmental damages would be tackled at time or at least minimized. For this end, and to avoid global environmental disasters and to ensure sustainable management policy, the basis of any exploration strategy of terrestrial or planetary resources should in my view be based on the following principle: 'reducing damages to minimum rather than to increasing benefits to maximum'.

\section{Disclosure: None.}

\section{References}

Abbott, B. P., et al. (2016). "Observation of Gravitational Waves from a Binary Black Hole Merger." Phys Rev Lett 116(6): 061102.

Crawford, I. A. and K. H. Joy (2014). "Lunar exploration: opening a window into the history and evolution of the inner Solar System." Philosophical transactions. Series A, Mathematical, physical, and engineering sciences 372(2024): 20130315.

Dittmar, M. (2014). "Development towards sustainability: How to judge past and proposed policies?" Science of The Total Environment 472: 282-288.

Fa, W. and Y.-Q. Jin (2007). "Quantitative estimation of helium-3 spatial distribution in the lunar regolith layer." Icarus 190(1): 15-23.

Huang, I. B., et al. (2011). "Multi-criteria decision analysis in environmental sciences: Ten years of applications and trends." Science of The Total Environment 409(19): 3578-3594.

Matsumoto, K., et al. (2006). "Japanese lunar exploration long-term plan." Acta Astronautica 59(1-5): 68-76.

Moustafa, K. (2016). "Oil, Earth mass and gravitational force." Sci Total Environ 548-549: 479-482.

Oleander, U. (2016). "Lunar mineral extraction to correct for potential loss of Earth mass caused by fossil fuel consumption." Sci Total Environ 557-558: 911.

Schaffer, A. M. (2008). "What do nations want from international collaboration for space exploration?" Space Policy 24(2): 95-103.

Williamson, M. (2005). "Lunar exploration and development--a sustainable model." Acta Astronaut 57(28): 161-166.

Zheng, Y., et al. (2008). "China's Lunar Exploration Program: Present and future." Planetary and Space Science 56(7): 881-886.

\footnotetext{
*Email: khaled.moustafa@arabixiv.org
} 